\title{
The Method of Complex Facility's Function Chart Comparison Produced by its Operator Expression and Application of Such a Method
}

\author{
Murat D. Adambayev ${ }^{1}$, Zhazira T. Julayeva ${ }^{1}$, Zhanibek E. Shukamanov', \\ Zhadyra D. Sadvakassova ${ }^{1}$, Gulmira S. Bayandina ${ }^{1}$, and Marzhan Spabekova ${ }^{1}$ \\ ${ }^{1}$ Kazakh Academy of Transport and Communications named after M. Tynyshpayev, Almaty, Kazakhstan.
}

ORCID: 0000-0003-3017-7133 (Zhanibek Shukamanov)

\begin{abstract}
The main component of the process of automation of any technological process is its identification (determination of the dynamic structure and parameters, usually represented as a transfer function Laplace. Currently, the effective methods of obtaining adequate mathematical models of the studied industrial control facilities are experimental methods; based on the processing of the results of both active experiment (removal of acceleration characteristics, obtaining frequency characteristics) and passive experiment with random realization of the input signals of a stochastic object on the studied channel with further processing of the experimental data associated with the solution of the Wiener-Hopf integral equation in the frequency, time domains or using operational amplifiers. All these methods make it possible to obtain the required mathematical models of complex technological processes (transfer function) in the form of an operator expression representing the ratio of power polynomials. The study of the obtained object model in the form of an operator expression cannot be used for modeling using operational amplifiers. The paper proposes we have developed a method that allows to produce an equivalent adequate function chart for the complex facility by the operator expression. Dividing all numerator and denominator additives of operator expression by the denominator component with the maximum degree of $\mathrm{a}_{0} \mathrm{~s}^{\mathrm{n}}$ can help to ensure the replacement of all differentiators with integrating factors. The resulting expression will transform into the flow chart. We have provided an example of structured modelling of complex industrial facility.
\end{abstract}

Keywords: identification, amplitude, phase, real and imaginary frequency characteristics, transfer function.

\section{INTRODUCTION}

A method is developed to obtain an equivalent adequate block diagram of the object by its known operator expression. Dividing all members of the numerator and denominator of the expression operator to the member in the denominator with the maximum degree $\left(a_{0} s^{n}\right)$ means to replace all differentiating links to an integrator. The resulting expression is converted to a block diagram. An example of structural modelling of a complex industrial facility is given.
The reading material provides a good description of the structured modelling (facility modelling to its known function chart) method which found a wide application. This case involves the function chart which is typically represented by a combination of typical dynamical elements with the transfer functions. For modelling purposes, functional diagram of a collection of facilities under study is produced by a modelling apparatus, and all necessary coefficients are estimated on the model, control equipment is connected, etc [1-3].

Producing a function chart of complex facility and obtaining its adequate mathematical model by theoretical methods seem to be impossible in identifying complex facilities for researching purposes [4-6]. To this end, in order to identify or define dynamic structure and parameters, new methods have been developed most recently as a result of processing the data observed [7-13].

The active experiment outcomes allow to obtain acceleration characteristics (facility's response to step input) which have one of the following types of sustainable linear objects: exponential, S-shaped or wave types. The shape of transient response curve determines the subject structure, and its dynamic parameters (transmission factors, response time and lateness) can be found as a result of processing transient response curve by special mathematical devices $[8,9]$.

The passive experiment outcomes (random samples of instance variables) allow to obtain an adequate mathematical model as a result of solving Wiener-Hopf integral equation in the frequency and time domains by operational (computing) amplifier [9, 11, 14-18].

\section{MATERIALS AND METHODS}

Typically, processing of active and passive experiment outcomes by the above methods allow us to obtain a mathematical model of the facility in the form of operator expression shown below [7,8,19-22]:

$$
\mathrm{W}(\mathrm{s})=\frac{\mathrm{b}_{0} s^{m}+b_{1} s^{m-1}+\ldots+b_{m-1} s+b_{m}}{a_{0} s^{n}+a_{1} s^{n-1}+\ldots+a_{n-1} s+a_{n}}=\frac{F_{1}(s)}{F_{2}(s)}
$$

Where: $F_{1}(s)$ - a polynomial of numerator with known coefficients $b_{j}(\mathrm{j}=0, \ldots, \mathrm{m}), \quad F_{2}(s)-$ a polynomial of denominator with known coefficients $a_{i}(\mathrm{i}=0, \ldots, \mathrm{n}), \mathrm{n}-$ operator expression order, $\mathrm{S}$ - Laplace operator. 
The modelling of the facility under study which has been obtained by the operator expression (1) is impossible to carry out due to consisting entirely of derivative elements which cannot be modelled due to being exposed to all types of electromagnetic interference.

Below is the method that allows to produce an absolutely equivalent function chart by an operator expression (1). Further, a structural modelling method widely used in the scientific research can also be applied.

In order to remove all derivative elements, (1) should be divided by $a_{0}, s^{n}$.

In order to reduce the scope of mathematical manipulation, let us consider a specific case (1), where $m=3 ; n=4$, meaning that

$$
\mathrm{W}(\mathrm{s})=\frac{\mathrm{b}_{0} s^{3}+b_{1} s^{2}+b_{2} s+b_{s}}{a_{0} s^{4}+a_{1} s^{3}+a_{2} s^{2}+a_{3} s+a_{4}}
$$

If we divide (2) by $a_{0} s^{4}$, we will further obtain

$$
\begin{aligned}
& W(s)=\frac{\frac{b_{0} s^{3}}{a_{0} s^{4}}+\frac{b_{1} s^{2}}{a_{0} s^{4}}+\frac{b_{2} s}{a_{0} s^{4}}+\frac{b_{s}}{a_{0} s^{4}}}{\frac{a_{0} s^{4}}{a_{0} s^{4}}+\frac{a_{1} s^{3}}{a_{0} s^{4}}+\frac{a_{2} s^{2}}{a_{0} s^{4}}+\frac{a_{3} s}{a_{0} s^{4}}+\frac{a_{4}}{a_{0} s^{4}}}= \\
& =\frac{\frac{1}{a_{0}}\left(\frac{b_{0}}{s}+\frac{b_{1}}{s_{2}}+\frac{b_{2}}{s_{3}}+\frac{b_{s}}{s^{4}}\right)}{1+\frac{1}{a_{0}}\left(\frac{a_{1}}{s}+\frac{a_{2}}{s^{2}}+\frac{a^{3}}{s^{3}}+\frac{a^{4}}{s^{4}}\right)}= \\
& =\frac{1}{1+\frac{1}{a_{0}}\left(\frac{a_{1}}{s}+\frac{a_{2}}{\mathrm{~s}^{2}}+\frac{a^{3}}{s^{3}}+\frac{a^{4}}{s^{4}}\right)} \times \frac{1}{1+\frac{1}{a_{0}}\left(\frac{a_{1}}{s}+\frac{a_{2}}{s^{2}}+\frac{a_{8}}{s^{3}}+\frac{a_{4}}{s^{4}}\right)} .
\end{aligned}
$$

A

$$
\times \underbrace{\frac{1}{a_{0}}}_{\mathrm{B}} \underbrace{\left(\frac{b_{0}}{s}+\frac{b_{1}}{s^{2}}+\frac{b_{2}}{s^{3}}+\frac{b_{s}}{s^{4}}\right)}=A \times B
$$

Let's consider the option of modelling the multiplier A in the (3) expression. To this end, let's examine the function chart shown in Figure 1, whose transfer function will be equal to [3]:

$$
W(s)=\frac{1}{1+W_{0}(s)}
$$

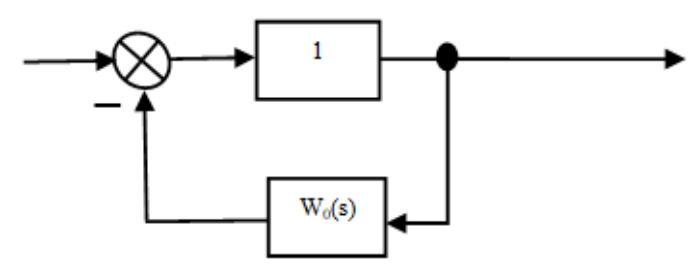

Fig.1. Flow chart

When comparing the expression (4) with the term A in the expression (3), we can conclude that the function chart of the term A corresponds to the flow chart, as shown in Figure 1, where the following condition is met:

$$
W_{0}(s)=\frac{1}{a_{0}}\left(\frac{a_{1}}{s}+\frac{a_{2}}{s^{2}}+\frac{a_{3}}{s^{3}}+\frac{a_{4}}{s^{4}}\right)
$$

The sample of function chart of the term A by meeting the condition (5) is shown in Figure 2.

A

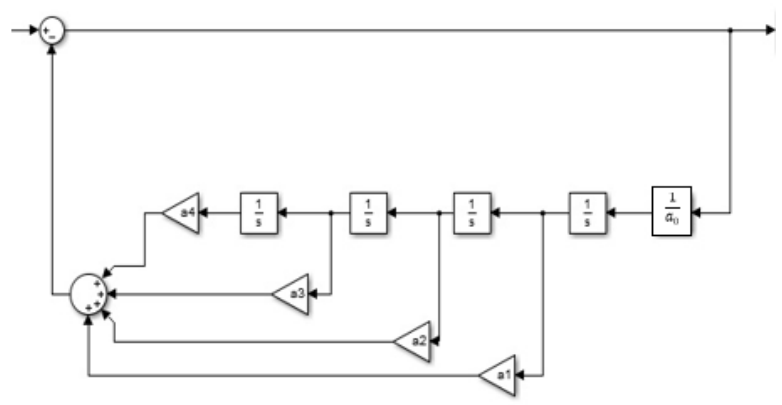

Fig. 2. Function chart of the term A

Let's produce a function chart of the term B by the expression (3) shown in Figure 3.

As the terms A and B are solved altogether, the flow charts of terms A and B need to be incorporated into one function chart and their output messages shall be multiplied by the divisionmultiplication unit (DMU). The resulting final function chart of expression (2) will be as shown in Figure 4.

B

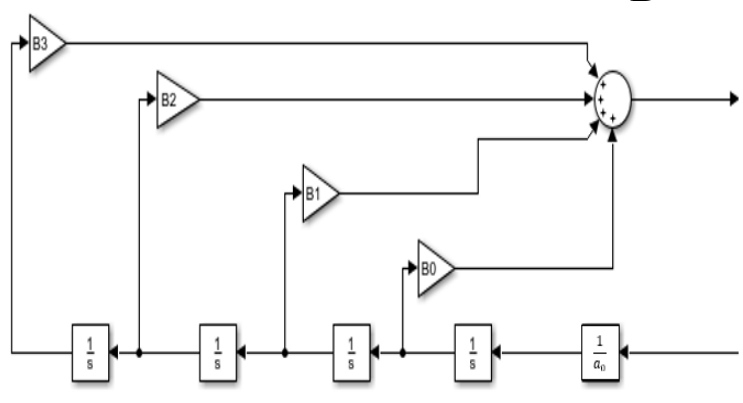

Fig. 3. The schematic structure of the term B 


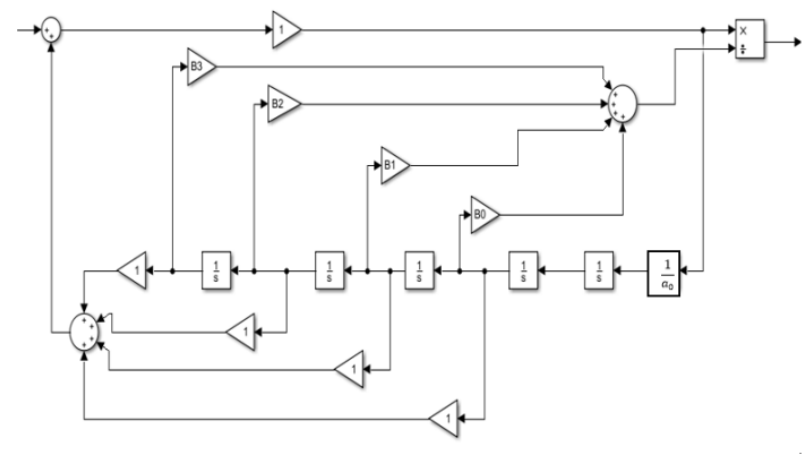

Fig. 4. The schematic structure of operator expression (2)

It follows that the operator expression (2) transforms into an equivalent function chart (see Figure 4) which can be easily set on the simulator and structured modelling method can easily be used to examine the entire facility under study.

By the method described above we have been able to obtain a function chart of the two-stage dry grinding using the Polisius-type bicameral grinding mills as major unites [4]. Below are the modelling results of this process which allowed to produce highly efficient systems of managing this complex process.

In order to define the parameters of two-stage grinding process and assess dynamic effects through the cross channels of the facility in the conditions of continually changing disturbances (such as ore grind ability that determines the value of circulating load will accordingly influence the number of material flowing though the facility, which would in turn impact the dynamic behaviour of the process), an analysis of function chart of the two-stage dry grinding process has been carried out using the operational (computing) amplifiers.

Transfer functions of all elements have been obtained as a result of processing measured curves of facility's transient behaviour during the step disturbance caused by the second stage original power supply and input power supply. The transfer functions of system elements are shown below:

$W_{n}(s)=K_{n}=1-$ Transfer function (TF) of rotary table;

$W_{2}(s)=\frac{e^{-4,9 s}}{1+5.1 s}-$ First stage transfer function;

$W_{2}(s)=\frac{e^{-2,9 s}}{1+3 s}-$ Second stage transfer function;

$W_{\text {э }}(s)=e^{-1 s}-$ Ladder-type trencher transfer function;

$W_{u}^{0}(s)=0,5$ - Damper-to-separator transfer function;

$W_{c}^{S}(s)=0,5 \div 0,9$ - Separator's transfer function through the channel: middling's penetration and extraction;

$W_{c}^{2}(s)=0,5 \div 0,1$ - Separator's transfer function through the channel: total input and output of end products;

$W_{u}^{I}(s)=0 \div 1-$ Damper transfer functions to the first «camera»;
$W_{u}^{I I}(s)=1 \div 0-$ transfer function to the second camera;

$W_{T_{1}}(s)=W_{T_{2}}(s)=W_{T_{3}}(s)=e^{-0,5 s}-$ Separator-to-grinding mill operating chambers inlet's pipeline transfer function.

The function chart has been produced to represent transfer functions. The inserted notations of system elements along with transfer functions of these elements that have been written in can boost the chart's visibility, therefore, not requiring any description of the chart's functioning.

The Figure 5 shows the linear model of the facility under study so as to set it on the operational amplifiers. As shown in the chart, $\mathrm{X}_{\mathrm{i}}$ - maximum permissible variable values required for calculating the $\alpha_{i}$ scale parameters. The Figure 6 shows the chart that represent numeric distribution of material flows under the steady-state operating conditions. It is also assumed that the system inlet is equal to the system outlet $\left(\mathrm{Q}_{\text {inlet }}=\mathrm{Q}_{\text {outlet }}\right.$ $=100$ c.u.), with the maximum circulating load being up to $400 \%$. In order to model the facility, the following are required:

- potentiometers - 23 pieces;

- integrators -8 pieces;

- summation unites - 2 pieces;

- inverters - 2 pieces;

- constant time-delay units (CTDU) - 5 pieces;

- scale elements - 11 units.

\section{RESULTS AND DISCUSSION}

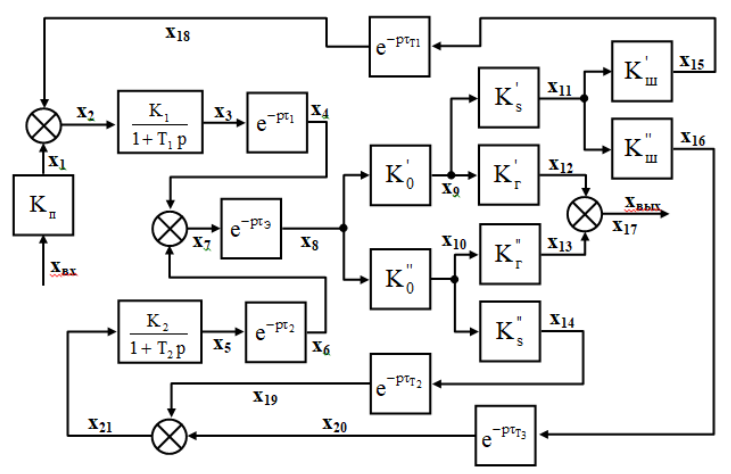

Fig. 5. Linear model of facility $\left(\mathrm{x}_{i}-\right.$ highest values of parameters)

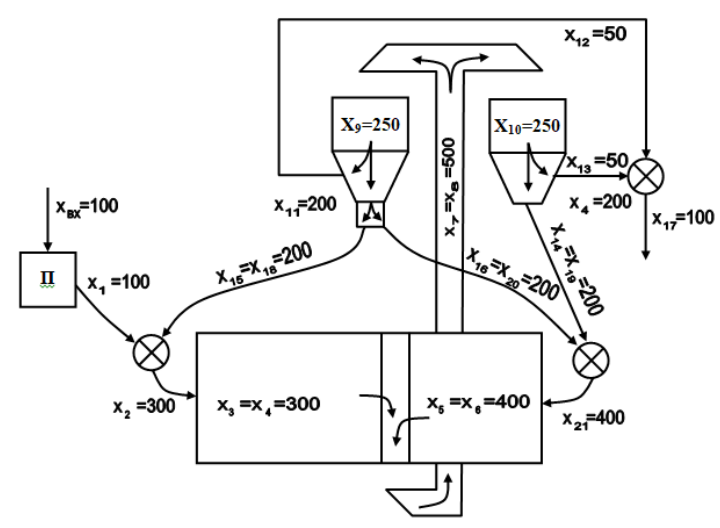

Fig. 6. Maximum permissible numeric distribution of material flows, provided that $\mathrm{S}$ equals to $400 \%$ 
The Figure 7 shows the functional diagram of a set of facility's linear model. All potentiometers, with the exception of $\alpha_{1}$, are automatic and set up to the compensation and threedigit selector scheme. The denomination of the latter on the distribution panel corresponds to AI-A90. $\alpha_{1}$ has been obtained through manual potentiometer P-76. The input signal is restricted to twenty volts. The circulating load fluctuation in the system are set at the range of $0 \div 400 \%$ of the feedstock value. The maximum variable values are between the points 7 and 8 which are restricted to 100 volts. The numbers of elements of the model that has been set are shown in the functional diagram that represents the models set inside the appropriate scheme elements. The scale elements and summation units have even notations, whereas integrators have uneven notations.

The four-rayed oscillograph is connected to the model through the selector which would allow to observe the variation in regimes in all model points where the values of varied coefficients change. The curves are recorded by the H320-5 type high-speed recorder using a special device.

The transport time-lags are set in the BPZ-2I constant time-lag units that have the УЭСВ-I type uninterrupted supply units.

Once the model has been tested, the following curves have been recorded:

1. At the outlet of coarse grinding chamber (after CTDU-I).

2. At the outlet of fine grinding chamber (after CTDU-2).

3. At the outlet of ladder-type trencher (after CTDU-3).

4. At the outlet of model (after 20 inverter).

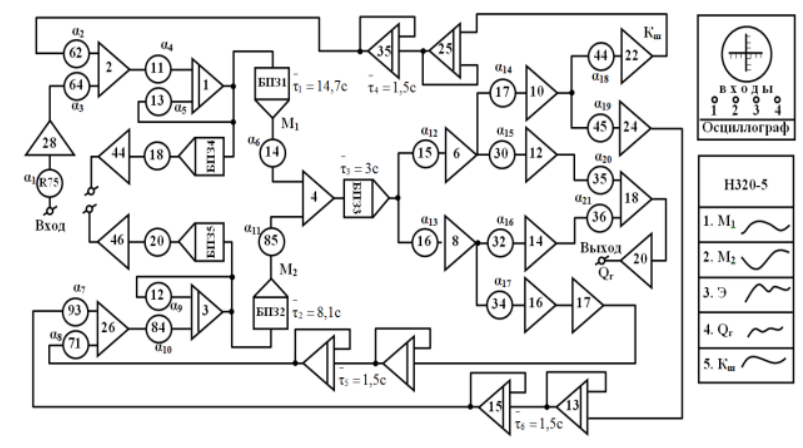

Fig. 7. Functional diagram of linear model set of two-stage

The Figure 8 shows the controlled variable variation curves during the step disturbance caused by the original power supply. When recording the transient behaviour curves, various coefficients to describe the ore grindability (number of circulating load) have been accepted. The curves are presented in two cases: when $\mathrm{K}_{\mathrm{s}}=0,5 ; 0,7$.

The transient behavour of coarse (IK) and fine grinding chambers (IIK) are of exponential curve shape with various amplification factors $(\mathrm{K})$ and response times $(\mathrm{T})$. For the first chamber, a response time coefficient is $\overline{\mathrm{T}}_{1}=16$ seconds where the pure transport time-lag is $\bar{\tau}_{1}=15$ seconds. The second chamber for the originial power supply (cross channel for this camera) has a considerably higher value of $\overline{\mathrm{T}}_{2}=24-25$ seconds and $\bar{\tau}_{2}=22-23$ seconds. When the disturbance is caused by the original power supply, acceleration factors and chamber time responses are dependent on the values of $\mathrm{K}_{\mathrm{s}}$ and $\mathrm{K}$.

The ladder-type trencher loading curve $\ni$ in the areas $\langle b-c »$ is sharp-bended. The area " $a-b$ " of this curve is determined by the variation in the first chamber loading, whereas an area " $c$ $d$ " determines the influence of the second chamber (see Figure 8 ). The curve of the end product number variation is proportional to the trencher loading, hence, it follows the trencher curve with a constant time-lag of 3 seconds (CTDU3).

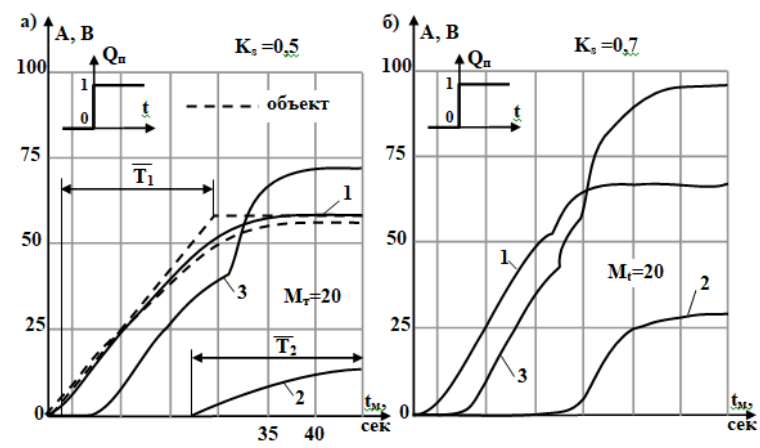

Fig. 8. Transient processes obtained in the model when the disturbance is caused by the original power supply $(1,2,3-$ first, second chambers and trenchers loading curves).

The Figure 9 shows these same curves, which, however, represent the harder ores that form the mass of sands $\left(\mathrm{K}_{\mathrm{s}}=\right.$ $0,7)$. As the Figure 8 shows, these curves differ from the previous ones in their shapes. The drastic increase in sands influences the shapes of transient behaviour curves of the first and second chambers.

The initial part of these curves is determined by the basic disturbance which is caused by original power supply, where, this disturbance results in the first chamber curve increasing by $70 \%$ of its established value, and the second chamber curve increasing by only $10 \%$ of its established value. The second part of curves is determined by the reverse positive relation, i.e. influence of sand load. This additional disturbance to a greater extent influences the degree of the second chamber loading $(85 \div 90 \%)$ and to a lesser extent influences the first chamber loading $(25 \div 30 \%)$ of established values of these parameters.

The trencher loading curve is sharp-bended twice which is caused by the basic and additional disturbances. The amplification factors and time responses of both cameras for the case of $K s=0,7$ have considerably increased as compared to $\mathrm{Ks}=0,5$.

The Figure 9 shows the curves with a step disturbance caused by the numeric distribution of sands loading between the chambers $\left(K_{\mathrm{II}}=\mathrm{var}\right)$. The Figure 8 shows the curves for the case of $K_{\mathrm{s}}=0,5$. The positive disturbance caused to the first camera corresponds to the negative disturbance caused to the second camera of the same range. The curves of transient 
process look almost the same as the $\mathrm{K}, \mathrm{T}, \tau$ curves. The trencher curve reflects the combined signal.

The failure at the initial area of the $\ni$ trencher loading curve is calculated by summing up two exponents with various transport time-lags.

The Figure 9 shows the equivalent curves for the case of $\mathrm{Ks}=$ 0,7 . The influence of basic disturbances caused by the sand loading and additional disturbances due to reverse causality during various transport time-lags are a precondition of damping oscillatory process in the ladder-type trencher.

Hence, variations in the values of original power supply determine the level of coarse grinding chamber loading and influence the fine grinding chamber loading levels through the cross channel of internal technological communication. For soft ores $\left(K_{s}=0,5\right)$, reverse causality does not have a considerable influence on the dynamic behaviour of the second chamber, while for the hard ores $\left(K_{s}=0,7\right)$, loop flows would otherwise cause additional disturbances which drastically affect the dynamic parameters of the system. The changes in $\mathrm{K}$ and $\mathrm{T}$ miller chambers are proportional to the ore grindability $\left(K_{s}\right)$. The changes in numeric distribution of sands drastically affect the loading levels of both chambers. When the disturbance is caused by the original power supply, the influence that the former has on the levels of fine grinding chamber loading through the cross channel is of soft monotonous nature with the low-time constant (which is influenced by the facility's filtering properties), hence, fine grinding chamber loading system should process such disturbances «smoothly» without using any compensating devices. When the fine grinding chamber loading levels are controlled by distributing the value of circulating load coming to this chamber, cross connection influencing the coarse grinding chamber loading level is strong and fluctuates drastically with high frequency.

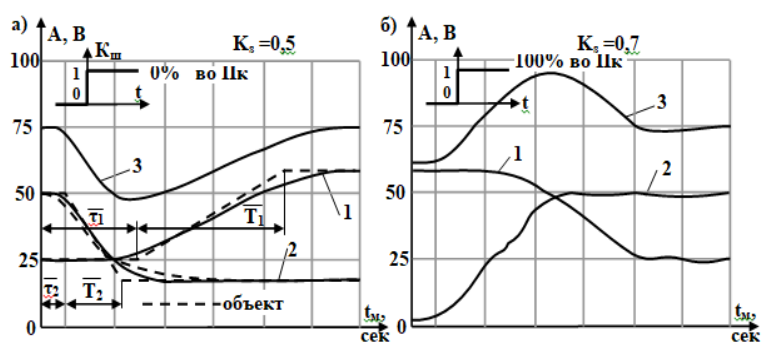

Fig. 9. Transient behaviour observed in the model, when disturbed by the sands loading (1, 2, 3- chambers and trenchers loading curves).

In other words, the coarse grinding chamber control system will be functioning in severe conditions, with the supply value being changed in a very wide range so as to maintain the controlled variable at the established level which would be linked to the huge fluctuations in the coarse grinding chambers loading levels. In some instances, (where the $\mathrm{K}_{\mathrm{s}}$ are high) this may lead to the loss of control. The solution to this problem may be the development of a special control system that would allow to compensate the influence of strong cross connections [23-24].

\section{CONCLUSION:}

1. The method is proposed to transform the operator expression of systems into an equivalent block diagram, which can be studied in depth on the modeling setup.

2. The results of modeling of a complex industrial control facility allow us to develop new highly efficient automatic control systems for such productions.

\section{REFERENCES}

[1] Adambayev M.D.. Increasing the efficiency of dry grinding process. Identification and automation. Saarbucken, Deutshland/Germany: LAP LAMBERT Academic Publishing, 2014, - p. 237.

[2] Balakirov V.S., Dudnikov E.T. \& Tsirlin A.M. Experimental observation of dynamic behaviour in industrial facilities. - M.: Energy, 1987 - p. 232.

[3] Adambayev M.D. Automatic dry ore dressing process management. Monography. - Almaty: Complex, 2013 p. 163.

[4] Venikov V. A., Venikov G. V. theory of similarity and modeling (in relation to problems of electric power industry): Ed. 4 R.-M.: Book house "Librokom", 2014440 S. (Classics of engineering: electrical power engineering).

[5] Baranov G. L., V. F. garcin Integrated simulation of electric power systems.- Kyiv: Naukova Dumka, 1979, 237c.

[6] Protosenya S. G., Kulish S. A., Azbel S. A. et al. Mathematical methods and models in planning and management of mining production - M.: Nebra, 1985, $288 \mathrm{c}$.

[7] Leung, L. Identification systems. Theory for the user: TRANS.. from English./Under the editorship of V. V. Tsypkina-M.: Science. Chapter ed. lit.- M.: Publishing house of KazGU. al-Farabi, 2012.

[8] Grop, D. Methods of identification systems. Per. from English. V. A. Vasilyev and V. I. Lopatin, ed. by E. I. Kineskog - M.: "Mir", 1979, 302s.

[9] Ribelin N. S., Chadeev V. M. Construction of models of production processes. - M.: "Energy", 1975, 376s.

[10] Adambaev M. D. Mathematical methods of identification. Textbook. - Almaty: KazNTU, 2012, $258 \mathrm{c}$.

[11] Adambaev M. D. Determination of the dynamic structure and parameters of industrial control facilities. Monograph.- Almaty: "TST-Company", 2010, 258c.

[12] Adambaev M. D. Theory and practice of technical experiment in electric power industry. Textbook.Almaty: KazNTU, 2013, 237c.

[13] 13.Adambaev M. D. Automatic control in the power industry. (Applied mathematics and identification). Textbook.- Almaty: KazNTU, 2015, 332c.

[14] M. D. Adambayev, Kuttybayev n, Auezov S. Dynamics of dry grinding in two-compartment separator. The 
scientific journal "Theoretical and practical solutions of mineral resources mining", 2015, London, Pp. 435-439.

[15] M. D. Adambayev, S. A. Kopaev, Auezov Alma., Kuttybayev A. Y. Research and development of software and hardware modules for testing technologies of rock mass blasting preparation. The scientific journal "Theoretical and practical solutions of mineral resources mining", 2015, London, Pp. 185-192.

[16] The adambayev M. D., Makenova B. Analysis of results of industrial tests of automatic control system on a cell load of two-chamber mills. Scientific journal "International scientific review", 2016 №2, Boston( USA), Pp. 44-48.

[17] K. Hartman, E. Lectures, V. Schaefer, etc. Planning of experiment in research of technological processes./ TRANS. S. him. under. edited by K. E. N. Leckage. M.: "Mir", 1977, 552c.

[18] Balakirov V. S., Dudnikov E. G., Tsilin S. M. Experimental determination of dynamic characteristics of industrial control objects. Moscow: Energiya, 1967, 232c.

[19] Rumshiskii L. Z. Mathematical processing of experimental results./ - M: the Main edition of physicomathematical. literature publishing house "Science", 1971, 192s.

[20] Hicks C. Basic principles of experiment planning. Translated from the English PD ed. - M.: "Mir" 1967$406 \mathrm{~S}$.

[21] Chapius P. L. The Experiment. Theory. Practice. - M.: Nauka, GL ed the physical and mathematical literature, 1977, $351 \mathrm{p}$

[22] M. D. Adambaev, Kuttybaev S. sh., Auezova S. Innovative technology of energy-intensive process of dry two-stage grinding. Bulletin of KazNTU named after K. I. Satpayev. - Almaty: 2015, No. 6. - P. 160165.

[23] The adambayev M. D., Auezov And Adambaeva Gautet and. Adequate discrete sampling method of random variables of two stage dry crushing progress and its stochastic equation. Thomson Reuters. Engineering for rural development, Jelgava, 23-25. 05.2018. http://www.tf.1lu.lv/conference/proceedings2018/, vol. 17, Pp. 994-1001, 2018.

[24] M. D. Adambayev, Kuttybayev n, Auezov S. Dynamics of dry grinding in two-compartment separator mills. Thomson Reuters. Collection of scientific works "New Developments in Mining Engineering: Theoretical and practical solutions of mineral resources mining". 30.09.2018 p.p 435-439/ publisher CRC Press Taylor\&Frsncis Group (Netherlands)

[25] M. D. Adambayev, S. A. Kopaev, Auezov Alma., Kuttybayev A. Y. Research and development of software and hardware modules for testing technologies of rock mass blasting preparation. Thomson Reuters. Collection of scientific works "New Developments in Mining Engineering: Theoretical and practical solutions of mineral resources mining". 30.09.2018 p.p 185-192/ publisher CRC Press Taylor\&Frsncis Group (Netherlands)22.

[26] Adambaev M. D., P. Shakerkhan metal from stone. The patent PRC No. 201120335636.3 No. document No. 00620590 from 09.01.2017 G.

[27] Device for continuous screening and grinding of mineral raw materials in p. Shakerkhan, M. D. Adambaeva. The patent PRC No. ZL201620480687. x. Date of authorisation 08.05.2016, certificate No. 2836025. 